\title{
Protocolo de avaliação e classificação de pacientes pediátri- cos conforme o grau de demanda da equipe de enfermagem
}

\author{
Protocol for the evaluation and classification of Pediatric patients \\ according to the nursing team demand level
}

\author{
Thalita Cristine Ramirez Duarte' $\bullet$ Everaldo Muniz de Oliveira² • Sérgio Luis Alves de Morais Júnior ${ }^{3}$
}

\begin{abstract}
RESUMO
Objetivou-se construir e apresentar um protocolo pediátrico conforme o grau de demanda da equipe de enfermagem em um Hospital Geral do estado de São Paulo.Trata-se de um estudo descritivo, exploratório e qualitativo, com aplicação de questionários e apresentação do protocolo através de uma aula expositiva e dialogada. Todos os participantes declararam ser importante avaliar e classificar os pacientes pediátricos, $76 \%$ não achou adequado o instrumento utilizado atualmente na instituição, $93 \%$ responderam que há a necessidade de um outro protocolo mais adequado às especificidades do paciente pediátrico, e apenas I4\% já conheciam o ICPP.A maioria dos participantes acham que o protocolo tem uma linguagem adequada, que pode beneficiar a prática clínica e que é de fácil aplicação, porém $10 \%$ não o acham viável. Conclui-se que para um dimensionamento adequado de pacientes pediátricos, é necessário um protocolo que contenha um instrumento que aborde as singularidades da criança e considere sua família como parte do cuidado. $O$ ICPP é um instrumento que utiliza uma linguagem adequada, beneficia a prática clínica, é de fácil aplicação, porém sua viabilidade depende de questões institucionais.
\end{abstract}

Palavras-chave: Protocolo;Dimensionamento de Pessoal;Enfermagem Pediátrica.

\begin{abstract}
The aim of this study was to construct and present a pediatric protocol according to the degree of demand of the nursing team in a general Hospital of the State of São Paulo. This is a descriptive, exploratory and qualitative study, with the application of questionnaires and presentation of the protocol through an expositive and dialogued class. All participants declared it important to evaluate and classify pediatric patients, $76 \%$ did not find adequate the instrument currently used in the institution, $93 \%$ answered that there is a need for another protocol more appropriate to specificities of the pediatric patient, and only 14\% already knew the ICPP. Most participants think that the protocol has an adequate language, which can benefit clinical practice and is easy to apply, but 10\% do not find it feasible. It is concluded that for an adequate dimensioning of pediatric patients, it is necessary a protocol that contains an instrument that addresses the singularities of the child and considers his family as part of the care. The ICPP is an instrument that uses appropriate language, benefits clinical practice, is easy to apply, but its viability depends on institutional issues.
\end{abstract}

Keywords: Protocol; Personnel Dimensioning; Pediatric Nursing. 


\section{INTRODUÇÃO}

Os serviços de saúde têm dentre os seus objetivos, a manutenção e a recuperação da saúde de indivíduos e populações, e para tal, é fundamental que se analisem questões gerenciais, como demanda, qualidade e segurança ofertada. Serviços de saúde com tecnologias mais adequadas, bem como estrutura física, insumos e recursos humanos têm mais condições de oferecer uma meIhor assistência aos seus pacientes ${ }^{(1)}$.

Os recursos humanos referem-se à equipe de trabalho que atua em determinado serviço de saúde. Considerando que o dimensionamento da equipe de enfermagem está diretamente relacionado com a qualidade e segurança da assistência prestada, é necessário que ele receba atenção especial por parte dos gestores de enfermagem ${ }^{(2,3)}$.

O dimensionamento é um processo sistemático que permite o equilíbrio entre a necessidade do número de profissionais de enfermagem e a demanda de cuidado, de forma a promover uma assistência de qualidade para um grupo de pacientes ${ }^{(2)}$.

Uma estratégia para auxiliar o dimensionamento são os Sistemas de Classificação de Pacientes (SCP), os quais permitem quantificar, avaliar e estimar a demanda de cuidados de enfermagem por grupos de pacientes, discriminando diferentes categorias de cuidado, segundo o grau de dependência da equipe de enfermagem. Tal estratégia, além de fundamentar e subsidiar a distribuição de pessoal de enfermagem, também auxilia no planejamento e na previsão de $\operatorname{custos}^{(3)}$.

O Conselho Federal de Enfermagem (COFEN), a partir da Resolução n. ${ }^{\circ}$ 543/2017, considera e estabelece parâmetros para o dimensionamento da equipe de enfermagem que subsidiam o planejamento, controle, regulação e avaliação das atividades assistenciais ${ }^{(4)}$.

As hospitalizações de pacientes pediátricos estão cada vez mais complexas e requerem estratégias voltadas aos aspectos psicossociais e familiares da criança ${ }^{(5)}$.

Atualmente, existem diversos instrumentos para $\circ$ SCP, e dentre eles, o mais adequado e sugerido para utilização pelo COFEN( ${ }^{(4)}$, é o Instrumento de Classificação de Pacientes Pediátricos (ICPP). É um instrumento validado, dividido em três domínios (família, paciente e procedimentos terapêuticos), composto por II indicadores e cada indicador tem quatro situações de dependência de cuidado graduadas de um a quatro pontos, de forma crescente conforme a demanda de cuidados. A partir disto, classificam-se cinco categorias de cuidado: Cuidados Mínimos, Cuidados Intermediários, Alta Dependência, Cuidados Semi-intensivos e Cuidados Intensivos ${ }^{(5)}$.

Para que os serviços de saúde utilizem um SCP de forma adequada, é necessária a criação de protocolos institucionais que orientem o preenchimento do instru- mento escolhido, delimite os locais onde será utilizado, normatize de acordo com a sua realidade e avalie os resultados dos mesmos, através de indicadores.

O protocolo deve ser entendido como o instrumento de auxílio que favorece a gestão do cuidado e, consequentemente, a assistência, pois ele permite o acesso à informações sistematizadas e tem como objetivo organizar, orientar e regulamentar o cuidado, facilitando assim, a conduta a ser tomada ${ }^{(6)}$.

Portanto, esta pesquisa visa contribuir com o processo gerencial de enfermeiras, subsidiando o dimensionamento da equipe de enfermagem, por meio do protocolo de avaliação e classificação de pacientes pediátricos conforme o grau de demanda da equipe de enfermagem, a fim de promover uma assistência segura e de qualidade.

O objetivo deste estudo foi construir e apresentar um protocolo de avaliação e classificação de pacientes pediátricos conforme o grau de demanda da equipe de enfermagem em um hospital geral do Estado de São Paulo.

\section{MÉTODO}

A pesquisa foi realizada em um hospital geral na Zona Sul do Município de São Paulo, com assistência médico -hospitalar de referência no atendimento de urgência e emergência de média e alta complexidades, e com administração público-privada. $O$ hospital é certificado como hospital de ensino e possui programas de estágios para as diversas áreas da saúde, além de especializações, residência médica e multiprofissional.

Compuseram os locais de estudo as unidades pediátricas de pronto-socorro infantil, internação pediátrica, internação cirúrgica pediátrica e a unidade de terapia intensiva pediátrica, que atende crianças de três dias aos 12 anos incompletos. O hospital conta com 286 leitos e destes, 62 são para setores pediátricos.

A amostra foi composta de 29 enfermeiros dos setores de cuidados pediátricos. Os critérios de inclusão foram, enfermeiras(os) ou residentes de enfermagem que atuem em alguma unidade pediátrica do hospital em questão há no mínimo três meses. Os critérios de exclusão foram, enfermeiras(os) ou residentes de enfermagem que não estivessem presentes no momento do estudo e que não aceitassem participar.

Todos os aspectos éticos foram respeitados conforme a Resolução n. ${ }^{\circ}$ 466/ 2 do Conselho Nacional de Saúde $\mathrm{CNS}^{(7)}$. A pesquisa foi apresentada para todos os participantes e foi explicada a importância da colaboração dos mesmos, tendo em vista os benefícios da compreensão do tema, sendo esclarecido a voluntariedade e liberdade de participação sem qualquer sanção ou prejuízo. Todos aqueles que aceitaram participar receberam o Termo de Consentimento Livre e Esclarecido (TCLE) e concorda- 
ram em assiná-lo. $O$ projeto de pesquisa foi aprovado pelo Comitê de Ética e Pesquisa da Universidade Santo Amaro (CEP/UNISA)sob o n. ${ }^{\circ} 2.661 .015$ e CAAE: 878559/8.0.0000.008I; e do hospital campo da pesquisa sob o n. ${ }^{\circ}$ 2.708.320 e CAAE: 878559 |8.0.300I.5447.

Pesquisa de caráter descritivo e exploratório com abordagem qualitativa $\mathrm{A}$ coleta de dados se deu em duas etapas, sendo a primeira, a aplicação de um questionário que verificou o conhecimento dos participantes sobre o tema, e levantou a necessidade de um instrumento adequado para a pediatria em comparação ao instrumento utilizado no hospital campo da pesquisa.

Após o término da primeira etapa foi iniciada uma aula expositiva dialogada, em queforam apresentados o protocolo e a orientação para sua aplicação. Em seguida, os participantes foram orientados a realizarem a avaliação e classificação de pacientes pediátricos a partir de estudos de casos diversos.

Essas estratégias pedagógicas foram escolhidas, pois permitem uma exposição do conteúdo juntamente com a participação dos sujeitos envolvidos, possibilitando que eles questionassem e interpretassem o objeto de estudo a partir do confronto com a sua realidade diária, além de lhes apresentar, através do estudo de casos, situações comuns, viabilizando uma minuciosa e objetiva avaliação do que lhe é proposto(8).

Por fim, foi realizada a segunda etapa da coleta de dados, com a aplicação de um novo questionário para avaliação do protocolo. Os dois questionários continham perguntas fechadas e possibilidades de resposta binária (sim ou não). $O$ primeiro questionário continha cinco perguntas e o segundo questionário continha quatro perguntas e um espaço para sugestões.

\section{RESULTADOS}

Em relação aos sujeitos participantes, a maioria era do sexo feminino, e apenas um dos participantes era do sexo masculino, o que corrobora com o perfil dos profissionais de enfermagem que é majoritariamente de mulheres $^{(9)}$.

Em relação à idade, a maioria tem entre 26 e 36 anos (45\%), seguido de pessoas entre 37 e 47 anos (4l\%), e por fim com idade menor ou igual a 25 anos (I4\%). Já no que diz respeito ao tempo de experiência dos profissionais em unidades pediátricas da determinada instituição, a maioria trabalha no hospital há mais de dez anos (3l\%), seguido por funcionários de um a 5 anos (28\%), e de cinco a dez anos e menos de um ano, ambos com $21 \%$ cada.

Como resposta ao primeiro questionário, todos os participantes (100\%) acreditam ser importante avaliar e classificar os pacientes pediátricos, tendo o escore resultante como um dos parâmetros para o dimensionamento da equipe de enfermagem. O hospital campo da pesquisa utiliza como SCP o instrumento criado por Fugulin e seus colaboradores ${ }^{(3)}$, porém, $76 \%$ dos participantes não o acham adequado para utilização em unidades pediátricas e, apenas $24 \%$ acha-o adequado, o que congrega com a orientação do COFEN que indica este protocolo como aplicável.

No que diz respeito à necessidade de um novo protocolo institucional, bem como a utilização de um instrumento mais adequado às especificidades do paciente pediátrico, $93 \%$ dos participantes responderam que há essa necessidade, e a maioria dos participantes (86\%) não conheciam outro instrumento com a mesma finalidade de avaliar e classificar o paciente para o dimensionamento diário da equipe, além do instrumento já utilizado no hospital. Apenas quatro participantes (I4\%) já conheciam o ICPP.

Como resposta à segunda etapa, obteve-se dados de avaliação do protocolo quanto à linguagem, benefícios da utilização na prática clínica, viabilidade e facilidade no preenchimento. Os resultados estão na tabela I.

A maioria dos participantes considera que o protocolo tem uma linguagem adequada, que pode beneficiar a prática clínica e que é de fácil aplicação, porém, $10 \%$ não o acham viável.

A sugestão que mais apareceu foi em relação à extensão do protocolo correlacionada à alta demanda de outras atividades requeridas pelo enfermeiro no diaadia associado à falta de recursos humanos no quadro da instituição. Também apareceram sugestões dizendo que o novo protocolo seria mais adequado para a prática clínica, solicitaram a disponibilidade do mesmo na forma impressa nas unidades e não apenas no sistema de informação e, que ele fosse implementado na instituição.

TABELA 1 - Questionário de avaliação do protocolo. São Paulo, SP, Brasil, 2018

\begin{tabular}{l|c|c}
\hline & SIM (\%) & NÃO (\%) \\
\hline Linguagem adequada & 100 & 0 \\
\hline Beneficiar a prática clínica & 100 & 10 \\
\hline Viável & 90 & 0 \\
\hline Fácil aplicação & 100 & 0 \\
\hline
\end{tabular}

Fonte: dados da pesquisa. 


\section{DISCUSSÃO}

O dimensionamento é um assunto que vem sendo estudado no mundo todo por representar uma atividade gerencial de grande importância para o enfermeiro ${ }^{(10)}$. 0 planejamento da assistência deve contar com uma avaliação tanto qualitativa como quantitativa de recursos humanos, e como subsídio, deve haver o uso de um SCP

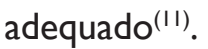

Evidencia-se que saber apenas o número de leitos ocupados não é um indicador seguro para estabelecer a quantidade de profissionais necessários para obter qualidade e segurança assistencial, é preciso uma caracterização da clientela que considere diversos fatores e necessidades individuais ${ }^{(12)}$.

No que se refere ao perfil do paciente pediátrico, existem diferenças inerentes à sua etapa de desenvolvimento, contudo, existem características comuns em relação à demanda de cuidado de enfermagem a serem consideradas $^{(13)}$.

Quando se caracteriza o paciente pediátrico, é notória a presença e envolvimento da família em seus cuidados. No Brasil, esse envolvimento vem sendo estudado na hospitalização da criança, tendo em vista o direito constituído pelo Estatuto da Criança e do Adolescente (ECA) que garante a presença de um familiar ou responsável legal acompanhando a criança ou o adolescente no período de sua internação ${ }^{(14,15)}$.

Isso vai de encontro com os princípios do Cuidado Centrado na Família, que tanto considera as necessidades de saúde da criança, como o bem-estar de sua família, além de incluí-la no planejamento dos cuida$\operatorname{dos}^{(16)}$. Para que isso ocorra, é necessário que a equipe proporcione meios de integrar a família nos cuidados, de forma que ela possa, dentro do possível, oferecer suporte à criança ${ }^{(14)}$.

Tendo em vista que o instrumento criado por Fugulin $^{(17)}$ não se aplica para unidades pediátricas e que o ICPP aborda não somente questões relacionadas ao pa- ciente e aos procedimentos terapêuticos, mas também inclui a família, acredita-se que ele seja um instrumento mais adequado, além de ser de prática e fácil aplicação(13).

Segundo estudo(18) que disserta sobre a utilização do ICCP em unidades pediátricas hospitalares, constatou-se que de fato ele permite um dimensionamento adequado que vai de encontro à resolução de dimensionamento de pessoal vigente. Porém, é importante destacar que o dimensionamento envolve diversos fatores que vão desde o quantitativo de recursos humanos disponíveis até questões como a filosofia e estrutura da instituição(13).

Sendo assim, uma instituição que não dispõe de recursos, não conseguirá realizar um dimensionamento adequado. De acordo com Fugulin ${ }^{(17)}$, diversas políticas e diretrizes que impõem contenção de gastos, não têm um aumento no quantitativo de profissionais que corresponda, e somado a isso, a enfermagem tem diversos fatores como falta investimento, precariedade de políticas de recursos humanos, remuneração insuficiente, carga de trabalho excessivo entre outros, que acabam afetando o rendimento e o desempenho desses profissionais, e por fim, a qualidade na assistência prestada.

\section{CONCLUSÃO}

Para um dimensionamento adequado de pacientes pediátricos, é necessário um protocolo que contenha um instrumento que aborde as singularidades da criança e considere sua família como parte do cuidado.

O ICPP é um instrumento que utiliza uma linguagem adequada, beneficia a prática clínica e é de fácil aplicação, porém, sua viabilidade depende de questões institucionais.

Espera-se com essa pesquisa, auxiliar no processo de implementação de um protocolo institucional que avalie e classifique o paciente pediátrico e sua família, considerando as suas especificidades, e assim, proporcionar dados que subsidiem um melhor dimensionamento da equipe de enfermagem e, por conseguinte, uma melhor assistência, segura e de qualidade. 


\section{REFERÊNCIAS}

I.Pinheiro RS, Escosteguy CC. Epidemiologia e serviços de Saúde. In: Medronho RA, Carvalho DM, Bloch KV, Luiz RR, Werneck GL. Epidemiologia. São Paulo: Atheneu; 2003. p. 36I-9.

2.FelliVEA, Peduzzi M. O trabalho gerencial em enfermagem. In: Kurcgant (coord). Gerenciamento em enfermagem. Rio de Janeiro: Guanabara Koogan, 2010. p. I- 12.

3.Fugullin FMT, Silva SHS, Shimizu HE, Campos FPF. Implantação do Sistema de Classificação de Pacientes na Unidade de Clínica Médica do Hospital Universitário da USP. RevMed HU-USP. 1994;4(I/2):63-8.

4. Conselho Federal de Enfermagem. Resolução COFEN n. ${ }^{\circ}$ 543/20 17. Parâmetros para o Dimensionamento do Quadro de Profissionais de Enfermagem nas Unidades Assistenciais das Instituições de Saúde e Assemelhados [Internet]. Brasília; 2017 [citado 2018 jan 15]. Disponível em: http://www.cofen. gov.br/resolucao-cofen-no-05272016_46348.html.

5.Dini AP, Guirardello EB. Sistema de classificação de pacientes pediátricos: aperfeiçoamento de um instrumento. Ver EscEnferm USP. 20 14;48(5):787-93.

6. Sousa RM. Atenção integral ao paciente onco-hematológico hospitalizado: proposta de um protocolo de cuidados de enfermagem. Rio de Janeiro. Dissertação [Mestrado Acadêmico em Ciências do Cuidado em Saúde] - Universidade Federal Fluminense; 2013.

7. Conselho Nacional de Saúde (BR). Resolução n. ${ }^{\circ} 466$, de 12 de dezembro de2012. Ética na pesquisa científica envolvendo seres humanos. Brasília - DF, 2012.

8.Mazzioni S. As estratégias utilizadas no processo de ensino -aprendizagem: concepções de alunos e professores de ciências contábeis. RevEletronAdm Tur. 2013; 2(1):93-109.

9. Oguisso T. Perfil do enfermeiro de unidades ambulatoriais do INAMPS no Brasil. Rev Esc Enf USP. 1990;24(I):77-92.

10. Rainio AK, Ohinmaa AE. Assessment of nursing management and utilization of nursing resourcer with the Rafaela patient classification system-case study from the general wards of one central hospital. J ClinNurs. 2005; I 4(6):674-84.

I I. Santos NC, Fugulin FMT. Construção e validação de instrumento paraidentificação das atividades de enfermagemem unidades pediátricas: subsídio paradeterminação da carga de trabalho. RevEscEnferm USP. 2013;47(5):I052-60.

12. Kurcgant P, Lima AFC, Prado C, Tronchin DMR, Fugulin FMT, Freitas GF etal. Gerenciamento em enfermagem. Rio de Janeiro: Guanabara Koogan; 2016. p.82-57.

13. Dini AP. Sistema de Classificação de Pacientes Pediátricos: construção e validação de um instrumento. Campinas. Dissertação [Mestrado em Enfermagem e Trabalho] - Faculdade de Ciências Médicas da Universidade Estadual deCampinas; 2007.

14. Albuquerque DB, Morais RCM, Macedo IF,Vieira RFC, Souza TV.A Família no cenário hospitalar pediátrico a partir da década de 1990: uma revisão integrativa. CogitareEnferm. 2013; 18(4):789-95.

15. Ministério da Ação Social (BR). Estatuto da criança e do adolescente. Lei n. ${ }^{\circ}$ 8.069, de 13 de junho de 1990. Dispõe sobre o Estatuto da Criança e do Adolescente, e dá outras providências. Diário Oficial da União, Brasília, DF, 1990 Jul 16. Seção I: I 3563.

16. Cruz AC, Angelo M. Cuidado centrado na família em pediatria: redefinindo os relacionamentos. CiencCuidSaude 201 I; 10(4):86I-865.

17. Fugulin FMT. Parâmetro oficiais para o dimensionamento de profissionais de enfermagem em instituições hospitalares: análise da Resolução COFEN n. ${ }^{\circ}$ 293/04. São Paulo. Tese [Livre-docência ao Departamento de Orientação Profissional] - Escola de Enfermagem da Universidade de São Paulo; 2010.

18. Gouveia MTO, Mendes MSC, Luz YPO, Silva GRF. Classificação de pacientes pediátricos em um hospital de ensino em Teresina. Revista da Rede de Enfermagem do Nordeste. 2010; I1:160-168. 